\title{
On Accuracy and Stability Analysis of the Reproducing Kernel Space Method for the Forced Duffing Equation
}

\author{
Bahram Asadi and Taher Lotfi \\ Department of Applied Mathematics, Hamedan Branch, Islamic Azad University, Hamadan, Iran \\ Correspondence should be addressed to Bahram Asadi; b.asadi@iauh.ac.ir
}

Received 29 July 2016; Accepted 7 September 2016

Academic Editor: Yuji Liu

Copyright ( 2016 B. Asadi and T. Lotfi. This is an open access article distributed under the Creative Commons Attribution License, which permits unrestricted use, distribution, and reproduction in any medium, provided the original work is properly cited.

It is attempted to provide the stability and convergence analysis of the reproducing kernel space method for solving the Duffing equation with with boundary integral conditions. We will prove that the reproducing space method is stable. Moreover, after introducing the method, it is shown that it has convergence order two.

\section{Introduction}

Reproducing kernel space method is a very powerful method for solving linear and nonlinear equation such as initial or boundary differential equation and integral equations [13]. This technique has been used not only for well-posed problems [4-6], but also for ill-posed problems [7]. In other words, the flexibility of choosing some tools in dealing with the given equation can be considered as the main reason for designing the solution method. Based on these features, one can use reproducing kernel space method efficiently to approximate the solution in any accuracy. In addition, it should be noted that, in fact, the applications of reproducing kernel Hilbert space method in the numerical analysis field are not new and on the other side possessing some of the wellknown advantages; for example [8-10],

(i) it is accurate, with needless effort to achieve the results,

(ii) it is possible to pick any point in the interval of integration and as well the approximate solutions and their derivatives will be applicable,

(iii) the method does not require discretization of the variables, and it is not affected by computation round off errors and one is not faced with necessity of large computer memory and time, (iv) it is of global nature in terms of the solutions obtained as well as its ability to solve other mathematical, physical, and engineering problems.

Duffing equation springs from modeling some different branches of sciences and engineerings such as chemical engineering, thermoelasticity, periodic orbit extraction, nonlinear mechanical oscillators, and prediction of diseases [1113]. To solve this equation, some variants of it have been investigated in recent years. One of them is due to Du and Cui, who applied an efficient method based on reproducing kernel space method (RKSM) [14]. Indeed, this technique is of great importance in solving linear and nonlinear equations [1]. Du and Cui used RKSM for solving the forced Duffing equation with boundary conditions [14] given by

$$
\begin{aligned}
& u^{\prime \prime}(x)+\sigma u^{\prime}(x)=f(x, u(x)), \quad 0<x<1, \sigma \neq 0, \\
& u(0)-\mu_{1} u^{\prime}(0)=\int_{0}^{1} h_{1}(x) u(x) d x, \\
& u(1)+\mu_{2} u^{\prime}(1)=\int_{0}^{1} h_{2}(x) u(x) d x,
\end{aligned}
$$

where $f:[0,1] \times R \rightarrow R, h_{i}: R \rightarrow R, i=1,2$, are continuous functions and $\mu_{i}, i=1,2$, are nonnegative constants.

To approximate the solution of the forced Duffing equation (1), however, based on our best knowledge, accuracy and stability have not been studied yet. In this work, it is attempted 
to study these issues. The rest of this paper is organised as follows.

Section 2 concerns reviewing some preliminaries. In Section 3, accuracy, convergence order, and stability are established. We confine ourselves to reporting the numerical implementation since they have been carried out in [14].

\section{Preliminaries}

In this section, we recall some basics which have been taken from [1]. We start with recalling the definition of $W_{2}^{m}[0,1]$ where $m$ is a positive integer. This space is the core of RKSM.

Definition 1. One has

$$
W_{2}^{m}[0,1]=\left\{u(x) \mid u^{(m-1)}(x)\right.
$$

is an absolutely continuous real function,

$$
\left.u^{(m)}(x) \in L^{2}[0,1]\right\} \text {. }
$$

The inner product and norm in $W_{2}^{m}[0,1]$ are defined, respectively, by

$$
\begin{aligned}
\langle u, v\rangle_{W_{2}^{m}}= & \sum_{k=0}^{m-1} u^{(k)}(0) v^{(k)}(0) \\
& +\int_{0}^{1} u^{(m)}(x) v^{(m)}(x) d x, \\
\|u\|_{W_{2}^{m}}= & \sqrt{\langle u, v\rangle_{W_{2}^{m}}},
\end{aligned}
$$

where $u, v \in W_{2}^{m}[0,1]$.

Also we need the following.

Definition $2 .{ }^{0} W_{2}^{3}[0,1]=\left\{u(x) \mid u^{\prime \prime}(x)\right.$ is an absolutely continuous real function; $u^{3}(x) \in L^{2}[0,1], u(0)-\mu_{1} u^{\prime}(0)=$ $\left.\int_{0}^{1} h_{1}(x) u(x) d x, u(1)+\mu_{2} u^{\prime}(1)=\int_{0}^{1} h_{2}(x) u(x) d x\right\}$.

The inner product and norm in ${ }^{0} W_{2}^{3}[0,1]$ are defined as mentioned above for any $u, v \in{ }^{0} W_{2}^{3}[0,1]$.

Definition 3 (reproducing kernel space, reproducing kernel). The function space $W_{2}^{m}[0,1]$ is called a reproducing kernel space if

$$
u(x)=\left\langle u(y), R_{y}(x)\right\rangle_{W_{2}^{m}}, \quad \forall u, R_{y} \in W_{2}^{m}[0,1] .
$$

Moreover, $R(x, y)$, or $R_{y}(x)$, is called the reproducing kernel.

Theorem 4 (see [15]). The reproducing kernel $R_{y}(x)$ in $W_{2}^{m}[0,1]$ is conjugate symmetric; that is, $R_{y}(x)=\overline{R_{x}(y)}$. It is also unique. Moreover, $R_{x}(x) \geq 0$, for each $x \in[0,1]$, and $R_{x}(x)=0$ if and only if $W_{2}^{m}[0,1]=\{0\}$.

It has been proven that the reproducing kernel space $W_{2}^{m}[0,1]$ is a complete space. Furthermore, for instance, the reproducing kernel of $W_{2}^{1}[0,1]$ and $W_{2}^{3}[0,1]$ is given [1], respectively, by

$$
\begin{aligned}
& R_{y}(x)= \begin{cases}1+x, & x \leq y, \\
1+y, \quad x>y,\end{cases} \\
& R_{y}(x) \\
& = \begin{cases}1+\frac{x^{5}}{120}+\frac{1}{12} x^{2} y^{2}(3+x)=x y\left(1-\frac{x^{4}}{24}\right), & x \leq y, \\
1+\frac{y^{5}}{120}+\frac{1}{12} x^{2} y^{2}(3+y)=x y\left(1-\frac{y^{4}}{24}\right), & x>y .\end{cases}
\end{aligned}
$$

\section{Accuracy and Convergence Analysis}

Here, we study the convergence order of the RKSM for solving (1). We will prove that this technique has convergence order two. Let $L u=u^{\prime \prime}+\sigma u^{\prime}$, where $L:{ }^{0} W_{2}^{3}[0,1] \rightarrow W_{2}^{1}[0,1]$; then, (1) can be written as follows:

$$
L u=f(x, u(x)), \quad 0<x<1,
$$

where $u(x) \in{ }^{0} W_{2}^{3}[0,1]$ and $f(x, u(x)) \in W_{2}^{1}[0,1]$. Therefore, $L$ is a linear and bounded operator on interval $[0,1]$.

To apply the RKSM, first of all, an orthogonal system of functions is constructed. Let $\varphi_{i}(x)=R_{x_{i}}(x)$, and then $\psi_{i}(x)=L^{*} \varphi_{i}(x)$, where $L^{*}$ is the conjugate operator of $L$. Consequently, because of the properties of the reproducing kernel, we have the following.

Lemma 5. One has $\left\langle u(x), \psi_{k}(x)\right\rangle_{0} W_{2}^{3}=L u\left(x_{k}\right), k=$ $1,2,3, \ldots$.

Proof. Consider

$$
\begin{aligned}
\left\langle u(x), \psi_{k}(x)\right\rangle_{0} W_{2}^{3} & =\left\langle u(x), L^{*} \varphi_{k}(x)\right\rangle_{0} W_{2}^{3} \\
& =\left\langle L u(x), \varphi_{k}(x)\right\rangle_{W_{2}^{1}} \\
& =\left\langle L u(x), R_{x_{k}}(x)\right\rangle_{W_{2}^{1}}=L u\left(x_{k}\right), \\
k & =1,2,3, \ldots
\end{aligned}
$$

If $\left\{x_{k}\right\}_{k=1}^{\infty}$ is dense on $[0,1]$, then $\left\{\psi_{i}(x)\right\}_{i=1}^{\infty}$ is a complete system of ${ }^{0} W_{2}^{3}[0,1]$ and $\psi_{i}(x)=\left.L R_{x}(y)\right|_{y=x_{i}}$ [1]. Applying the well-known Gram-Schmidt process, an orthonormal system, for example, $\left\{\bar{\psi}_{k}(x)\right\}_{k=1}^{\infty}$ in ${ }^{0} W_{2}^{3}[0,1]$, is generated from $\left\{\psi_{k}(x)\right\}_{k=1}^{\infty}$ by

$$
\bar{\psi}_{i}(x)=\sum_{k=1}^{i} \beta_{i k} \psi_{k}(x),
$$

where $\beta_{i k}$ are the orthogonalization coefficients, $\beta_{i j}>0, i=$ $1,2,3, \ldots$.

According to [14], we have the following solution method. 
Theorem 6 (see [14]). If $\left\{x_{k}\right\}_{k=1}^{\infty}$ is dense on $[0,1]$, and $u(x) \in$ ${ }^{0} W_{2}^{3}[0,1]$ is the solution of $(1)$, then

$$
u(x)=\sum_{i=1}^{\infty} A_{i} \bar{\psi}_{i}(x)
$$

where $A_{i}=\sum_{k=1}^{i} \beta_{i k} f\left(x_{k}, u\left(x_{k}\right)\right), i=1,2,3, \ldots$

It is worth nothing that when $f$ is nonlinear, this method can not be used directly in action. Therefore, an iterative modified version of it has been introduced as follows.

Theorem 7 (see [14]). If $\left\{x_{k}\right\}_{k=1}^{\infty}$ is dense on $[0,1], u_{0}(x) \in$ ${ }^{0} W_{2}^{3}[0,1]$ is given, and $u(x) \in{ }^{0} W_{2}^{3}[0,1]$ is the solution of $(1)$, then

$$
u(x)=\sum_{i=1}^{\infty} B_{i} \psi_{i}(x), \quad u_{0} \in{ }^{0} W_{2}^{3}[0,1],
$$

where $B_{i}=\sum_{k=1}^{i} \beta_{i k} f\left(x_{k}, u_{k-1}\left(x_{k}\right)\right), i=1,2,3, \ldots$.

To obtain the approximate solution $u_{n}(x)$, a proper truncated series of $u(x)$ is used by

$$
\begin{array}{r}
u_{n}(x)=\sum_{i=1}^{n} A_{i} \bar{\psi}_{i}(x), \\
\text { or } u_{n}(x)=\sum_{i=1}^{n} B_{i} \bar{\psi}_{i}(x),
\end{array}
$$

where $A_{i}$ or $B_{i}$ are given as before.

The main contribution of [14] says that, under the given conditions (see Theorems 3.1 and 3.2 in [14]), the approximate solution $u_{n}(x)$ converges to the exact solution $u(x)$. Nevertheless, applying the numerical results by $\mathrm{Du}$ and Cui in [14], they converge quadratically. Surprisingly, this fact has been neither stated nor proved already. So, we state and prove it formally here. First, we need the following lemma.

Lemma 8. Let the conditions of the Theorem 6 be held. Moreover, suppose that $f$ is independent of $u$. Then,

$$
L u_{n}\left(x_{k}\right)=f\left(x_{k}\right), \quad k=1,2,3, \ldots, n .
$$

Proof. Because of the properties of reproducing kernel definition and assumptions, we have

$$
\begin{aligned}
L u_{n}\left(x_{k}\right) & =\left\langle L u_{n}(x), R_{x_{k}}(x)\right\rangle_{W_{2}^{1}} \\
& =\left\langle L u_{n}(x), \varphi_{k}(x)\right\rangle_{W_{2}^{1}} \\
& =\left\langle u_{n}(x), L^{*} \varphi_{k}(x)\right\rangle_{{ }^{0} W_{2}^{3}} \\
& =\left\langle u_{n}(x), \psi_{k}(x)\right\rangle_{{ } W_{2}^{3}} \\
& =\left\langle\sum_{i=1}^{n} A_{i} \bar{\psi}_{i}(x), \psi_{k}(x)\right\rangle_{{ }^{0} W_{2}^{3}} \\
& =\sum_{i=1}^{n} A_{i}\left\langle\bar{\psi}_{i}(x), \psi_{k}(x)\right\rangle_{0} W_{2}^{3} .
\end{aligned}
$$

Using this relation with orthonormality and definition of $\left\{\bar{\psi}_{j}\right\}$, we have

$$
\begin{aligned}
A_{n} & =\sum_{i=1}^{n} A_{i}\left\langle\bar{\psi}_{i}, \bar{\psi}_{j}\right\rangle_{0} W_{2}^{3}=\sum_{i=1}^{n} A_{i}\left\langle\bar{\psi}_{i}, \sum_{j=1}^{n} \beta_{n j} \psi_{j}\right\rangle_{{ }^{0} W_{2}^{3}} \\
& =\sum_{j=1}^{n} \beta_{n j} L u_{n}\left(x_{j}\right) .
\end{aligned}
$$

On the other hand, based on the definition of $A_{n}$ in Theorem 6 and the assumption that $f$ is independent of $u$, we have $A_{n}=\sum_{j=1}^{n} \beta_{n j} f\left(x_{j}\right)$. It is now sufficient to equate right-hand sides of these two relations for definition of $A_{n}$, when $n$ varies. Then, the proof is complete.

In what follows, we provide a priori and a posteriori error estimations.

Theorem 9. Suppose that $u_{n}(x)$ and $u(x)$ are the approximate and the exact solution of (1), generated by RKSM in Theorem 6, $r_{n}(x)=L u(x)-L u_{n}(x)$, and $e_{n}(x)=u(x)-u_{n}(x)$. If $0=x_{1}<$ $x_{2}<\cdots<x_{n}=1, h_{i}=x_{i+1}-x_{i}, i=1,2, \ldots, n-1$, and $h=\max h_{i}$, then

$$
\begin{aligned}
& \left\|r_{n}\right\|_{\infty}=O\left(h^{2}\right), \\
& \left\|e_{n}\right\|_{\infty}=O\left(h^{2}\right),
\end{aligned}
$$

where $\left\|e_{n}\right\|_{\infty}=\max _{x \in[0,1]}\left|e_{n}(x)\right|$.

Proof. By Lemma 10, we have $r_{n}\left(x_{j}\right)=0, j=1,2, \ldots, n$. If $p_{1}(x)$ interpolates $r_{n}(x)$ at nodes $x_{k}$ and $x_{k+1}$, then $p_{1}(x)=0$. Therefore,

$$
\begin{aligned}
r_{n}(x) & =r_{n}(x)-p_{1}(x) \\
& =\frac{\left(x-x_{k}\right)\left(x-x_{k+1}\right)}{2} f^{\prime \prime}\left(\eta_{k}\right),
\end{aligned}
$$

where $\eta_{k}$ is between $x_{k}$ and $x_{k+1}$. Thus, we have $\left|r_{n}(x)\right| \leq$ $M h^{2}$, for some constant $M$ and $h=\max \left\{h_{k}, h_{k+1}\right\}$. This completes the first assertion. Furthermore, since $L$ is a bounded linear operator, it is invertible, and, therefore, $e_{n}(x)=L^{-1} r_{n}(x)$ and the second estimation follows. ing.

Very similar to the above argument, we have the follow-

Lemma 10. Let the conditions of Theorem 7 be held. Then,

$$
L u_{n}\left(x_{k}\right)=f\left(x_{k}, u_{k-1}\left(x_{k}\right)\right), \quad k=1,2,3, \ldots, n .
$$

Similar to Theorem 9, we can conclude the following.

Theorem 11. Suppose that $u_{n}(x)$ and $u(x)$ are the approximate and the exact solution of (1), generated by RKSM in Theorem 7 , 
$r_{n}(x)=L u(x)-L u_{n}(x)$, and $e_{n}(x)=u(x)-u_{n}(x)$. If $0=x_{1}<$ $x_{2}<\cdots<x_{n}=1, h_{i}=x_{i+1}-x_{i}, i=1,2, \ldots, n-1$, and $h=\max h_{i}$, then

$$
\begin{aligned}
\left\|r_{n}\right\|_{\infty} & =O\left(h^{2}\right), \\
\left\|e_{n}\right\|_{\infty} & =O\left(h^{2}\right),
\end{aligned}
$$

where $\left\|e_{n}\right\|_{\infty}=\max _{x \in[0,1]}\left|e_{n}(x)\right|$.

Now, we deal with the stability of RKHS method for the solution of $L u(x)=f(x, u(x))$, where the operator $L$ is given in (6). For this purpose, suppose that the right-hand side has $\varepsilon>0$ perturbation. We indicate variation of the approximate solution is bounded by a constant multiple of $\varepsilon$. In other words, approximate solution depends continuously on the right-hand side. We need the following.

Lemma 12 (see [15]). If $u(x) \in{ }^{0} W_{2}^{3}[0,1]$, then there is a constant $c$ such that

$$
\left\|u^{(k)}\right\|_{\infty} \leq c\|u\|_{0} W_{2}^{3}, \quad 0 \leq k \leq 2 .
$$

Theorem 13. Consider the problem $\operatorname{Lu}(x)=f(x, u(x))$, which has a unique solution, and $L:{ }^{0} W_{2}^{3}[0,1] \rightarrow W_{2}^{1}[0,1]$ is bounded linear. Then, the approximate solution obtained from RKHS method (9) is stable.

Proof. Suppose that $u_{n}(x)$ is the approximate solution of the abovementioned equation obtained from RKHS method; that is,

$$
u_{n}(x)=\sum_{i=1}^{n} \sum_{k=1}^{i} \beta_{i k} f\left(x_{k}, u\left(x_{k}\right)\right) \bar{\psi}_{i}(x)
$$

where $x_{k} \in[0,1], \bar{\psi}_{i}(x)$, and $\beta_{i k}$ are orthonormal bases and coefficient obtained from Gram-Schmidt orthogonalization process. Moreover, suppose that $v(x)$ is the approximate solution of $\operatorname{Lu}(x)=f(x, u(x))+\varepsilon(x)$, where $\varepsilon(x)>0$ and is bounded. We prove that there exists constant $\delta>0$ such that $\left\|v_{n}-u_{n}\right\|_{\infty}<\delta$. According to the definition of $u_{n}(x)$ and $v_{n}(x)$, we have

$$
\begin{aligned}
v_{n}(x) & -u_{n}(x) \\
= & \sum_{i=0}^{n} \sum_{k=0}^{i} \beta_{i k}\left(f\left(x_{k}, u\left(x_{k}\right)\right)+\varepsilon\left(x_{k}\right)\right) \bar{\psi}_{i}(x) \\
& \quad-\sum_{i=0}^{n} \sum_{k=0}^{i} \beta_{i k} f\left(x_{k}, u\left(x_{k}\right)\right) \bar{\psi}_{i}(x) \\
= & \sum_{i=0}^{n} \sum_{k=0}^{i} \beta_{i k} \varepsilon\left(x_{k}\right) \bar{\psi}_{i}(x) .
\end{aligned}
$$

On the other hand, $L^{-1}$ exists and $L^{-1} \varepsilon(x) \in{ }^{0} W_{2}^{3}[0,1]$. Therefore,

$$
\begin{aligned}
L^{-1} \varepsilon(x) & =\sum_{i=0}^{n} \sum_{k=0}^{i} \beta_{i k}\left\langle L^{-1} \varepsilon(x), \psi_{k}(x)\right\rangle_{{ }_{0} W_{2}^{3}} \bar{\psi}_{i}(x) \\
& =\sum_{i=0}^{n} \sum_{k=0}^{i} \beta_{i k}\left\langle\varepsilon(x),\left(L^{-1}\right)^{*} \psi_{k}(x)\right\rangle_{{ }_{0} W_{2}^{3}} \bar{\psi}_{i}(x) \\
& =\sum_{i=0}^{n} \sum_{k=0}^{i} \beta_{i k} \varepsilon\left(x_{k}\right) \bar{\psi}_{i}(x) .
\end{aligned}
$$

Since the right-hand sides of relations (21) and (22) are equal, then

$$
v_{n}(x)-u_{n}(x)=L^{-1} \varepsilon(x)
$$

Since $L^{-1}$ is continuous on $[0,1]$, it is bounded and we have

$$
\left\|v_{n}(x)-u_{n}(x)\right\|_{0} W_{2}^{3}=\left\|L^{-1}\right\|_{0} W_{2}^{3}\|\mathcal{E}(x)\|_{0} W_{2}^{3} \cdot
$$

Hence, with $M=\left\|L^{-1}\right\|_{0} W_{2}^{3}$, we conclude that $\left\|v_{n}-u_{n}\right\|_{0} W_{2}^{3} \leq$ $M\|\varepsilon(x)\|_{0} W_{2}^{3}$. Based on Lemma 12, $\left\|v_{n}-u_{n}\right\|_{\infty} \leq c M \varepsilon$ and therefore $\delta=c M \varepsilon$.

Similarly, we have the following theorem.

Theorem 14. Consider the problem $L u(x)=f(x, u(x))$, which has a unique solution, and $L:{ }^{0} W_{2}^{3}[0,1] \rightarrow W_{2}^{1}[0,1]$ is bounded and linear. Then, the approximate solution obtained from RKHS method (10) is stable.

\section{Competing Interests}

The authors declare that there is no conflict of interests regarding the publication of this paper.

\section{Acknowledgments}

Also, the authors acknowledge Hamedan Branch of Isalmic Azad University for their support during conducting this research.

\section{References}

[1] M. Cui and Y. Lin, Nonlinear Numerical Analysis in the Reproducing Kernel Space, Nova, 2009.

[2] Z. Chen and Z. Chen, "The exact solution of system of linear operator equations in reproducing kernel spaces," Applied Mathematics and Computation, vol. 203, no. 1, pp. 56-61, 2008.

[3] F. Z. Geng and S. P. Qian, "Reproducing kernel method for singularly perturbed turning point problems having twin boundary layers," Applied Mathematics Letters, vol. 26, no. 10, pp. 998-1004, 2013.

[4] F. Geng and M. Cui, "A reproducing kernel method for solving nonlocal fractional boundary value problems," Applied Mathematics Letters, vol. 25, no. 5, pp. 818-823, 2012. 
[5] F. Z. Geng and S. P. Qian, "A new reproducing kernel method for linear nonlocal boundary value problems," Applied Mathematics and Computation, vol. 248, pp. 421-425, 2014.

[6] R. Ketabchi, R. Mokhtari, and E. Babolian, "Some error estimates for solving Volterra integral equations by using the reproducing kernel method," Journal of Computational and Applied Mathematics, vol. 273, pp. 245-250, 2015.

[7] H. Du and M. Cui, "Approximate solution of the Fredholm integral equation of the first kind in a reproducing kernel Hilbert space," Applied Mathematics Letters, vol. 21, no. 6, pp. 617-623, 2008.

[8] O. Abu Arqub, "Adaptation of reproducing kernel algorithm for solving fuzzy Fredholm-Volterra integrodifferential equations," Neural Computing and Applications, 2015.

[9] O. Abu Arqub, M. AL-Smadi, S. Momani, and T. Hayat, "Numerical solutions of fuzzy differential equations using reproducing kernel Hilbert space method," Soft Computing, vol. 20, no. 8, pp. 3283-3302, 2016.

[10] O. Abu Arqub, M. Al-Smadi, S. Momani, and T. Hayat, "Application of reproducing kernel algorithm for solving second-order, two-point fuzzy boundary value problems," Soft Computing, 2016.

[11] B. Ahmad and B. S. Alghamdi, "Approximation of solutions of the nonlinear Duffing equation involving both integral and non-integral forcing terms with separated boundary conditions," Computer Physics Communications, vol. 179, no. 6, pp. 409-416, 2008.

[12] M. Momeni, N. Jamshidi, A. Barari, and D. D. Ganji, "Application of He's energy balance method to Duffing-harmonic oscillators," International Journal of Computer Mathematics, vol. 88, no. 1, pp. 135-144, 2011.

[13] S. A. Khuri and S. Xie, "On the numerical verification of the asymptotic expansion of Duffing's equation," International Journal of Computer Mathematics, vol. 72, no. 3, pp. 325-330, 1999.

[14] J. Du and M. Cui, "Solving the forced Duffing equation with integral boundary conditions in the reproducing kernel space," International Journal of Computer Mathematics, vol. 87, no. 9, pp. 2088-2100, 2010.

[15] X. Y. Li and B. Y. Wu, "Error estimation for the reproducing kernel method to solve linear boundary value problems," Journal of Computational and Applied Mathematics, vol. 243, no. 1, pp. 10-15, 2013. 


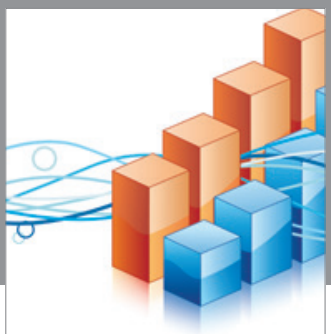

Advances in

Operations Research

vatem alat4

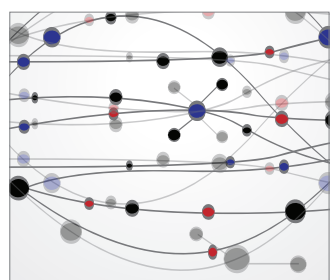

\section{The Scientific} World Journal
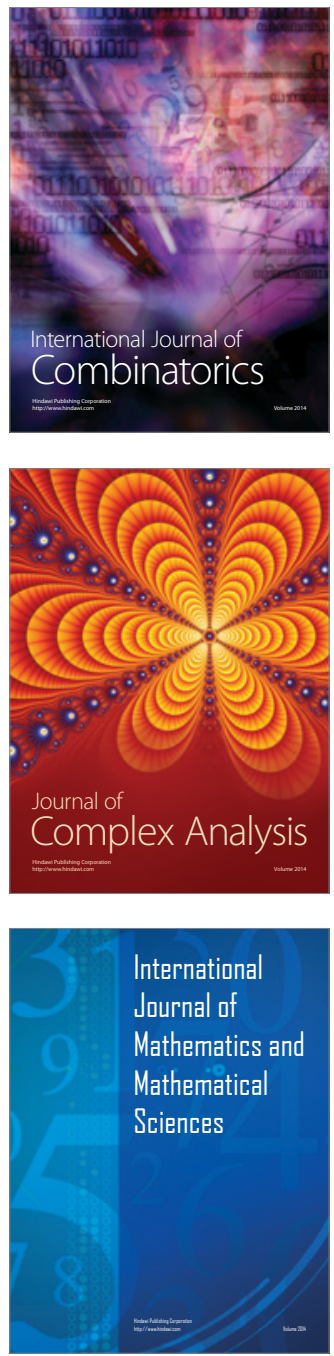
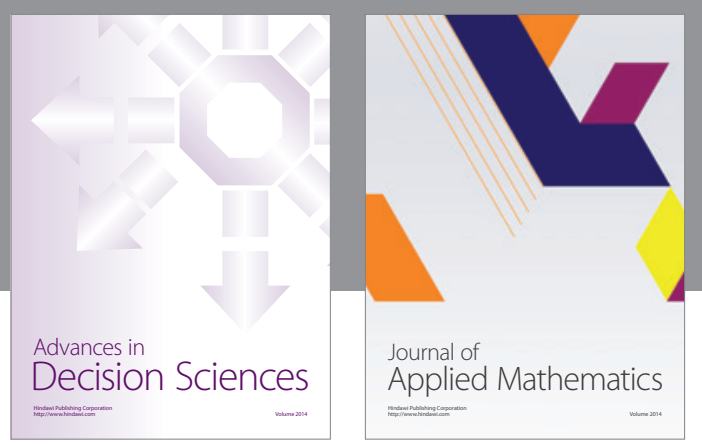

Algebra

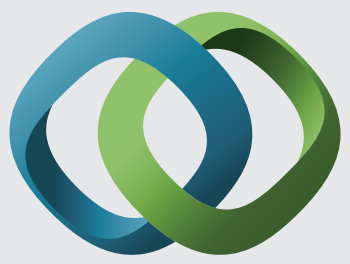

\section{Hindawi}

Submit your manuscripts at

http://www.hindawi.com
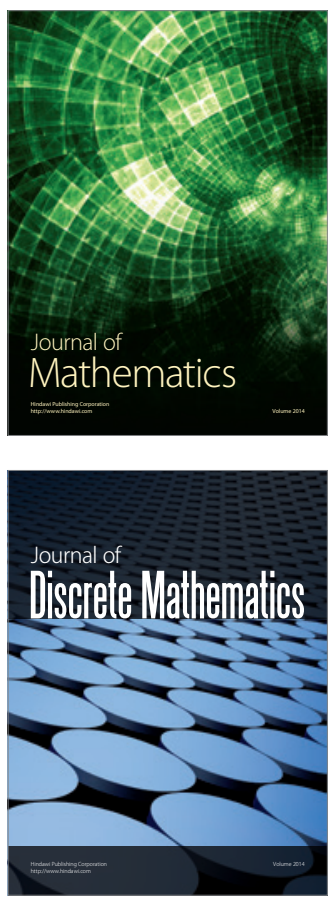

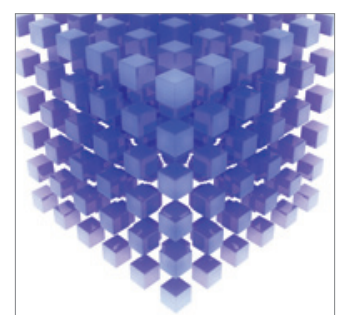

Mathematical Problems in Engineering
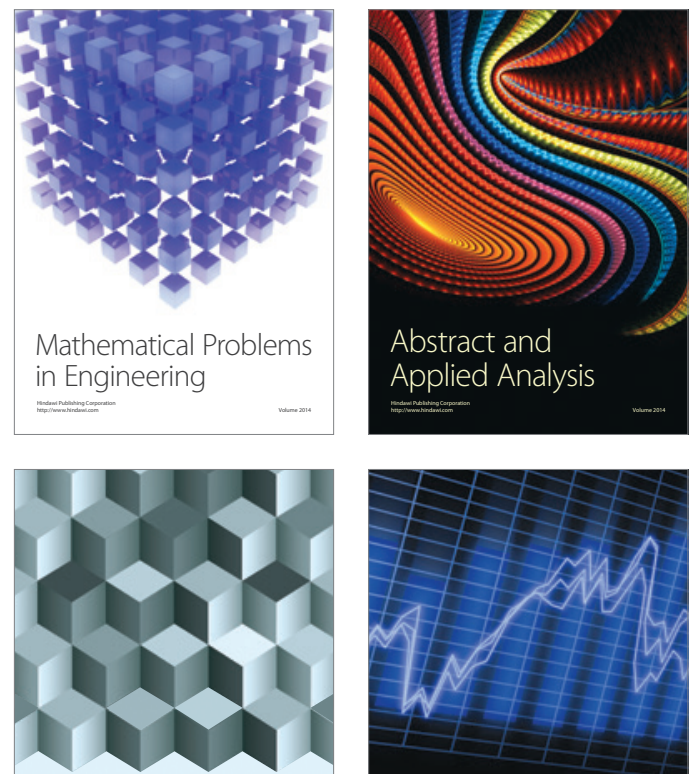

Journal of

Function Spaces

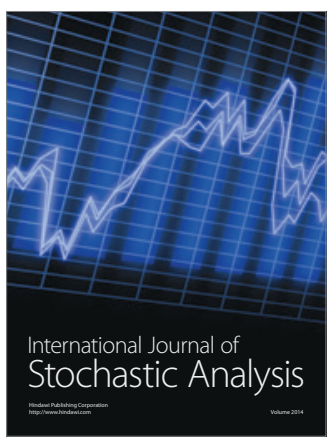

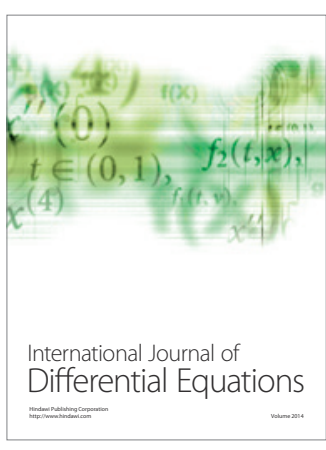
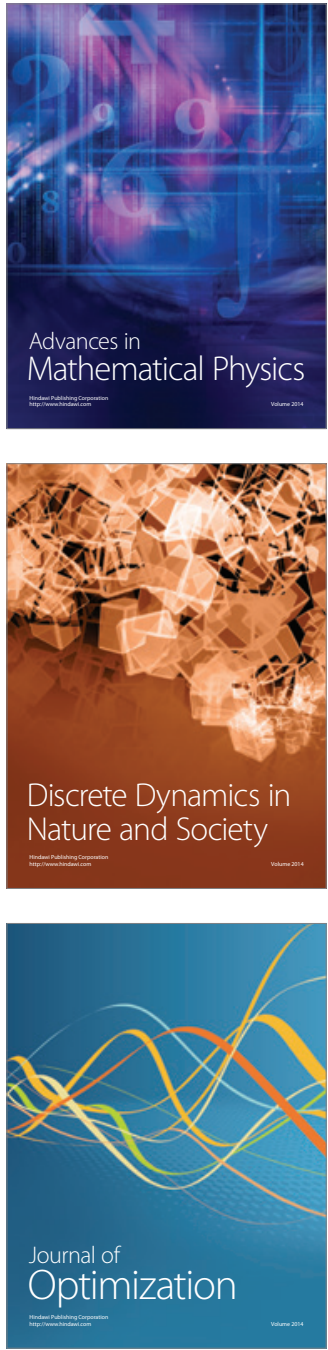